\title{
33 ON THE RE-CONFIGURABILITY DESIGN OF PARALLEL MACHINE TOOLS
}

\author{
Dan Zhang \\ Faculty of Engineering and Applied Science, \\ University of Ontario Institute of Technology \\ Oshawa, Ontario, $\mathrm{LIH} 7 \mathrm{~K} 4$, Canada \\ Dan.Zhang@uoit.ca
}

\begin{abstract}
The ongoing globalisation and international competition of manufacturing is requiring manufacturers to become more agile to efficiently use production resources. The ability to rapidly reconfigure production processes allows rapid ramp-up and robustness to adapt to unexpected events. In this paper, the reconfigurable machine tools and its characteristics is discussed in detail. Firstly, the basic modules of reconfigurable parallel machine tools are introduced. Then the criteria to form the reconfigurable machine are discussed. Some conceptual examples are illustrated and one case study is conducted.
\end{abstract}

\section{INTRODUCTION}

As the main component of reconfigurable manufacturing systems, the reconfigurable machine tools are machine tools that are built from machine modules [1]. A machine module could be an actuator, a joint, a link, a tool holder or a spindle. These modules are designed to be easily reconfigured to accommodate new machining requirements. Therefore, the reconfigurable machine tools have the characteristics of modularity, convertibility, integrate-ability, customisation, flexibility and costeffectiveness.

The main objective of reconfigurable machine tool design is reconfigurable components that are reliable and cost-effective. It is noted that machine design will be conducted at the component level.

Some researchers already focused on the RMT study. Koren and Kota [2] received a patent for an RMT where the type, location, and number of spindles could be adjusted in response to changing product requirements. Landers et al.[3,4] presented an overview of reconfigurable machine tools and their characteristics,. Zhang et al. [5-7] developed a generic kinetostatic model for RMT stiffness analysis and design optimisation. Yigit and Ulsoy [8] discussed the vibration isolation of RMTs and proposed different isolation strategies depending on the RMT requirement.

As described by Koren et al. [9], the modular construction of Parallel Kinematic Machines (PKMs), allow them to be used as a special class of reconfigurable machine tools, consist of simple and identical modules that can be configured into different machines. Parallel kinematic machines can be also used as particular modules for machining lines. 
According to Jovane [10], reconfigurable parallel kinematic machines should have:

- Modular and reconfigurable structure with the possibility to change the total DOF,

- Light structure and high dynamic performances, as a standard PKM,

- Possibility to use innovative materials in the machine design

- Possibility to use fast actuators such as linear motors

- Due to the intrinsic modularity, a DOF decoupling is recommended

- Low complexity in the kinematic chains.

\section{BASIC COMPONENTS}

There are 4 basic types of joints, they are: revolute joint, Hook (universal) joint, Spherical joint and prismatic joint (Figure 1). One can develop the reconfigurable parallel machine by combination of those joints and links.
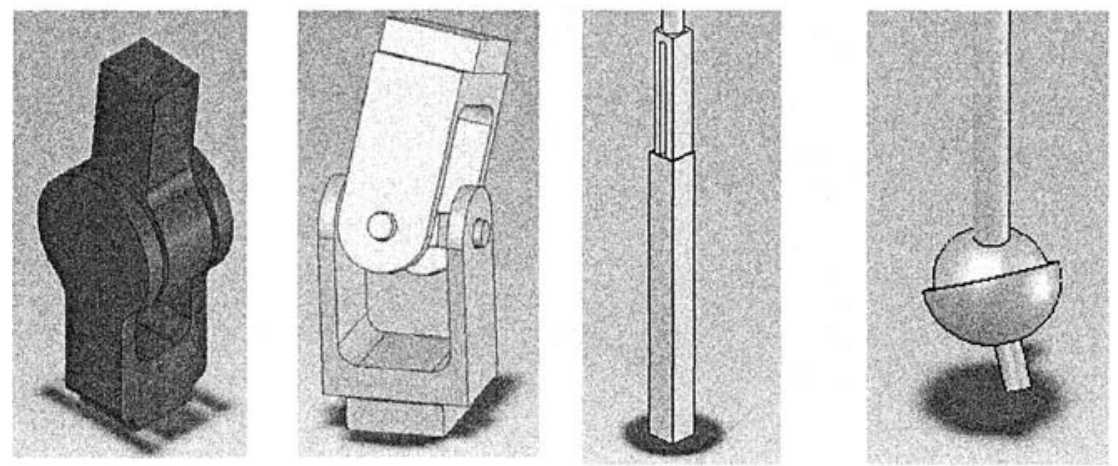

Figure 1 - Basic modular components

\section{DESIGN CRITERIA}

In order to design the reconfigurable parallel kinematic machines properly, there are several criteria that need to be followed with, here are some major issues: the degree of freedom of the architecture; design constraints used for most promising typology selection and kinetostatic model for stiffness analysis and design optimisation.

\subsection{Mobility}

A preliminary evaluation of the mobility of a kinematic chain can be found from the Chebychev-Grübler-Kutzbach formula.

$$
M=d(n-g-1)+\sum_{i=1}^{g} f_{i}
$$

where $M$ denotes the mobility or the system DOF, $d$ is the order of the system ( $d=3$ for planar motion, and $d=6$ for spatial motion), $n$ is the number of the links including the frames, $g$ is the number of joints, and $f_{i}$ is the number of DOFs for the $i$ th joint. 


\subsection{Constraints}

In order to ensure the required motions (i.e., 5-DOF between the machine tool and the workpiece), the DOFs distribution numbers and the type of motions for each leg should be properly selected. Each leg can be facilitated with spherical, prismatic, Hooke and revolute joints.

The legs used in machine tools must be simple and practical to implement. For the sake of the simplicity and dexterity of mechanism, we prefer to use "spherical" pairs as the joints between link and the moving platform for those legs with more than 3 DOFs. Since the serially connected revolute joints easily lead to singularities and the manufacturability is difficult, so we forgo their use for cases where more than 2 revolute joints connected in series.

The study is based on fully-parallel mechanisms, but one can add legs (with 6dof) to keep the structure symmetric. For the shape of the platforms, one should avoid the use of regular polygon, since it may lead to singularities.

\section{SOME EXAMPLES}

In Figures $2-4$, we proposed a series of reconfigurable parallel mechanisms which consist of $\mathrm{n}$ identical actuated legs with six degrees of freedom and one passive leg with $\mathrm{n}$ degrees of freedom connecting the platform and the base. The degree of freedom of the mechanism is dependent on the passive leg's degree of freedom. One can improve the rigidity of this type of mechanism through optimization of the link rigidities to reach a maximized global stiffness and precision. Finally, this series of mechanisms have the characteristics of reproduction since they have identical actuated legs, thus, the entire mechanism essentially consists of repeated parts, offering price benefits for manufacturing, assembling and purchasing. It acts as a kind of reconfigurable parallel machines, based on a number of identical active kinematic chains plus a passive central leg, to compensate torsion. The reconfiguration process consists on the possibility to change the total DOF by adding or removing active struts, and, consequently constraining the passive leg.
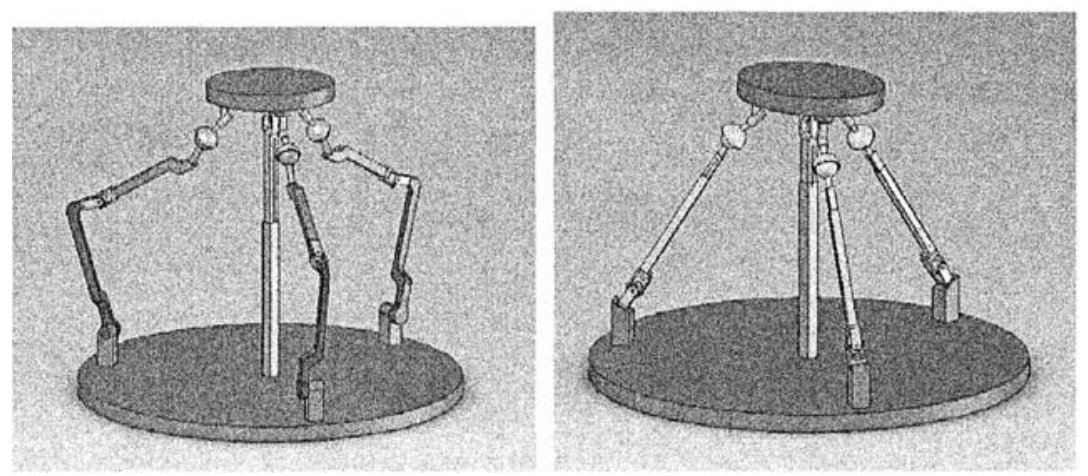

Figure 2 - Reconfigurable parallel robot with 3DOF 

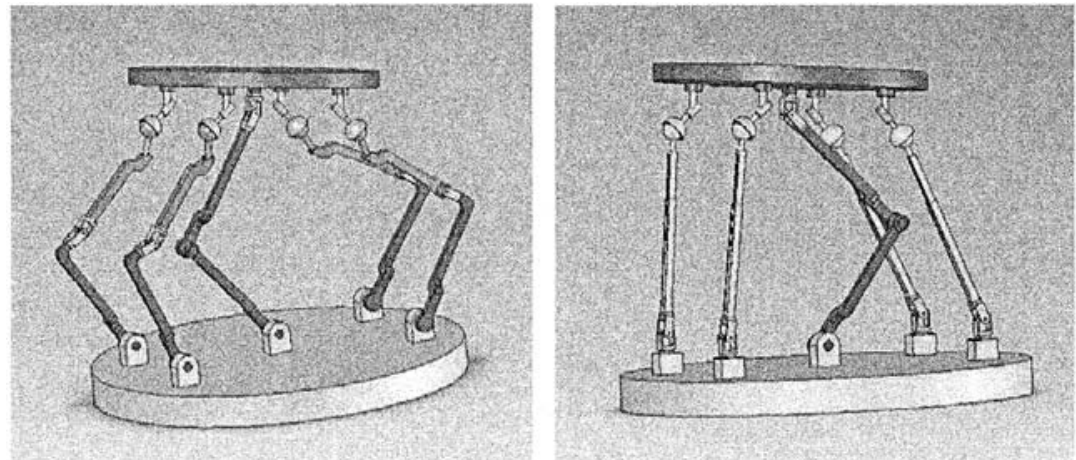

Figure 3 - Reconfigurable Parallel Robot with 4DOF
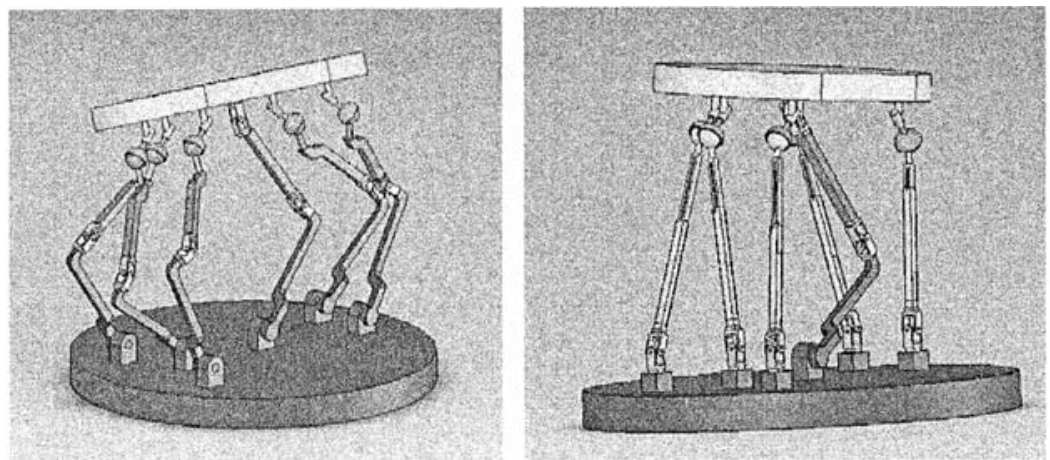

Figure 4 - Reconfigurable Parallel Robot with 5DOF

\section{CASE STUDY}

We take a 3DOF reconfigurable parallel robot (Figure 2) with prismatic actuators as an example. The Cartesian coordinates of the platform are given by the position of point $\mathrm{P}$ with respect to the fixed frame, and the orientation of the platform (orientation of frame $\mathrm{P}-\mathrm{X}^{\prime} \mathrm{Y}^{\prime} \mathrm{Z}^{\prime}$ with respect to the fixed frame), represented by the joint angles of the fourth leg $\theta_{42} \cdot \theta_{43}$ (central leg) or by the matrix $Q$. 


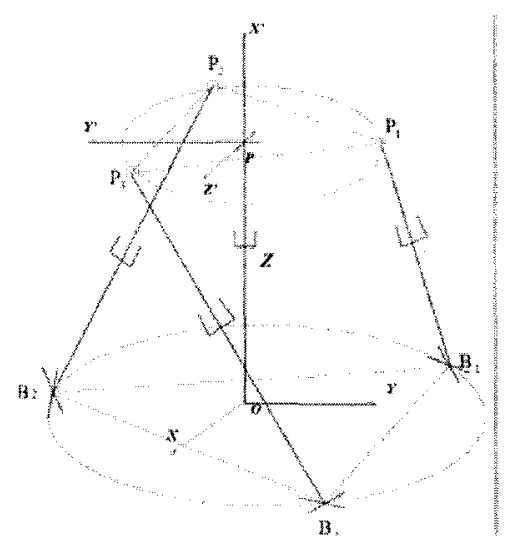

Figure 5 - Schematic Representation of Reconfigurable Parallel Robot with 3DOF

If the coordinates of the point $P_{i}$ in the moving reference frame are represented

with $\left(x_{i}^{\prime}, y_{i}^{\prime}, z_{i}^{\prime}\right)$ and the coordinates of the point $B_{i}$ in the fixed frame are represented by vector $b_{i}$, then for $i=1, \ldots, 4$, we have

$$
\mathbf{p}_{i}=\left[\begin{array}{l}
x_{i} \\
y_{i} \\
z_{i}
\end{array}\right] \mathbf{r}_{i}^{\prime}=\left[\begin{array}{l}
x_{i}^{\prime} \\
y_{i}^{\prime} \\
z_{i}^{\prime}
\end{array}\right] \mathbf{p}=\left[\begin{array}{l}
x \\
y \\
z
\end{array}\right] \mathbf{b}_{i}=\left[\begin{array}{l}
b_{i x} \\
b_{i y} \\
b_{i z}
\end{array}\right]
$$

where $\mathbf{p}_{i}$ is the position vector of point $P_{j}$ expressed in the fixed coordinate frame whose coordinates are defined as $x_{i}, y_{i}, z_{i}$, and $\mathbf{r}_{i}$ is the position vector of point $P_{i}$ expressed in the moving coordinate frame, and $\mathbf{p}$ is the position vector of point $P$ expressed in the fixed frame as defined above,

We can then write

$$
\mathbf{p}_{\mathrm{i}}=\mathbf{p}+\mathbf{Q} \mathbf{r}_{\mathrm{i}}
$$

where $\mathbf{Q}$ is the rotation matrix corresponding to the orientation of the platform of the manipulator with respect to the base coordinate frame.

Subtracting vector $\mathbf{b}_{\mathfrak{i}}$ from both sides of eq.(3), we obtain

$$
\mathbf{p}_{\mathrm{i}}-\mathbf{b}_{\mathrm{i}}=\mathbf{p}+\mathbf{Q} \mathbf{r}_{\mathrm{I}}-\mathbf{b}_{\mathrm{i}}
$$

then taking the Euclidean norm of both sides of eq.(4), we derive

$$
\rho_{\mathrm{i}}^{2}=\left(\mathbf{p}_{\mathrm{i}}-\mathbf{b}_{\mathrm{i}}\right)^{\mathrm{T}}\left(\mathbf{p}_{\mathrm{i}}-\mathbf{b}_{\mathrm{i}}\right)
$$

where $\rho$ is the length of the ith leg, i.e. the value of the ith joint coordinate. The solution of the 3-dof platform is therefore completed. 


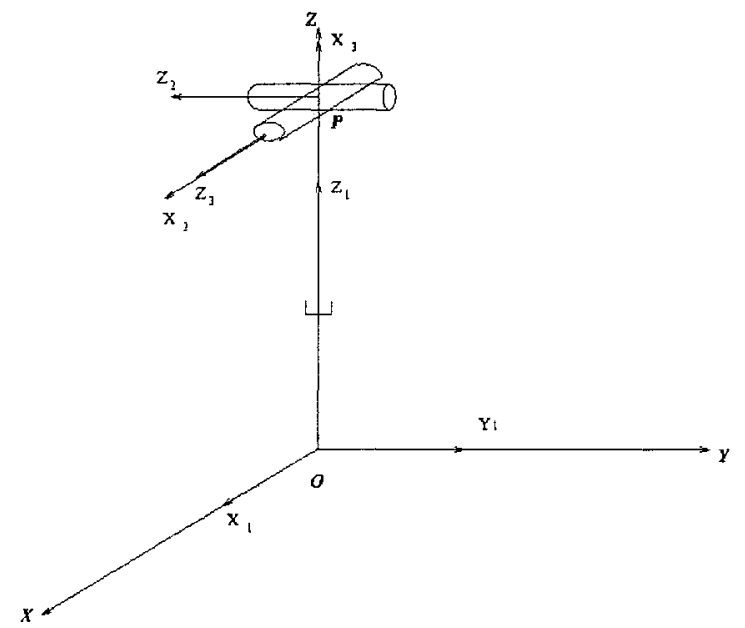

Figure 6 - The Central Leg of Reconfigurable Parallel Robot with 3DOF

Figure 6 illustrates the configuration of the passive leg. From the Figure 6, we can obtain the Denavit-Hartenberg parameters as in Table 1 ,

Tablel: The DH parameters for the passive constraining leg with rigid links

\begin{tabular}{|c|c|c|c|c|}
\hline $\mathrm{i}$ & $\mathrm{a}_{\mathrm{i}}$ & $\mathrm{b}_{\mathrm{i}}$ & $\alpha_{\mathrm{i}}$ & $\theta_{\mathrm{i}}$ \\
\hline 0 & 0 & 0 & 0 & 0 \\
\hline 1 & 0 & $\mathrm{Z}$ & 90 & 0 \\
\hline 2 & 0 & 0 & 90 & $\theta_{42}$ \\
\hline 3 & 0 & 0 & 0 & $\theta_{43}$ \\
\hline
\end{tabular}

with the Denavit-Hartenberg parameters above, we can obtain the kinematic model.

The kinematic chain can be taken as a serial manipulator, the kinematics of the serial manipulators comprise the study of the relations between joint variables and Cartesian variables. A Hooke joint can be replaced by two orthogonal revolute joints in this case.

For the passive kinematic chain, we have the velocity equation

$$
\mathbf{J}_{4} \theta_{4}=\mathbf{t}
$$

where

$$
\dot{\boldsymbol{\theta}}_{4}=\left[\begin{array}{lll}
\dot{\rho} & \dot{\theta}_{42} & \dot{\theta}_{43}
\end{array}\right]^{T}
$$

and the Jacobian matrix of the 4 th leg of the mechanism $\mathbf{J}_{4}$ can be expressed as

$$
\mathbf{J}_{4}=\left[\begin{array}{ccc}
0 & \mathbf{e}_{42} & \mathbf{e}_{43} \\
\mathbf{e}_{41} & \mathbf{e}_{42} \times \mathbf{r}_{42} & \mathbf{e}_{43} \times \mathbf{r}_{43}
\end{array}\right]
$$


Differentiating eq.(5) with respect to time, we have the velocity equation as

$$
\mathbf{A t}=\mathbf{B} \dot{\boldsymbol{\rho}}
$$

where vectors $\dot{\rho}$ and $\mathbf{t}$ are the joint velocity and the twist of the platform defined as

$$
\begin{aligned}
& \dot{\boldsymbol{\rho}}=\left[\begin{array}{lll}
\dot{\rho}_{1} & \dot{\rho}_{2} & \dot{\rho}_{3}
\end{array}\right]^{T} \\
& \mathbf{t}=\left[\begin{array}{ll}
\boldsymbol{\omega}^{T} & \dot{\mathbf{p}}^{T}
\end{array}\right]^{T}
\end{aligned}
$$

According the principle of virtual work, we have

$$
\boldsymbol{\tau}^{T} \dot{\boldsymbol{\rho}}=\mathbf{w}^{T} \mathbf{t}
$$

where $\tau$ is a vector of the actuator forces applied at each actuated joint and $w$ is the wrench (torque and force) applied to the platform and where it is assumed that no gravitational forces act on any of the intermediate links.

We have $\mathbf{w}=\left[\begin{array}{ll}\mathbf{n}^{T} & \mathbf{f}^{T}\end{array}\right]^{T}$ where $\mathbf{n}$ and $\mathbf{f}$ are respectively the external torque and force applied to the platform.

Regarding eq.(9) and substituting it in eq. (12), we obtain

$$
\boldsymbol{\tau}^{T} \mathbf{B}^{-1} \mathbf{A t}=\mathbf{w}^{T} \mathbf{t}
$$

Now, substituting eq.(6) into eq.(13), we have

$$
\boldsymbol{\tau}^{T} \mathbf{B}^{-1} \mathbf{A J}_{4} \dot{\boldsymbol{\theta}}_{4}=\mathbf{w}^{T} \mathbf{J}_{4} \dot{\boldsymbol{\theta}}_{4}
$$

The latter equation must be satisfied for arbitrary values of $\dot{\boldsymbol{\theta}}_{4}$ and hence we can write

$$
\left(\mathbf{A} \mathbf{J}_{4}\right)^{T} \mathbf{B}^{-T} \tau=\mathbf{J}^{T}{ }_{4} \mathbf{w}
$$

The latter equation relates the actuator forces to the Cartesian wrench, $\mathbf{w}$, applied at the end-effector in static mode. Since all links are assumed rigid, the compliance of the mechanism will be induced solely by the compliance of the actuators. An actuator compliance matrix $\mathbf{C}$ is therefore defined as

$$
C \tau=\Delta \rho
$$

where $\tau$ is the vector of actuated joint forces and $\Delta \rho$ is the induced joint displacement. Matrix $\mathrm{C}$ is a $(3 \times 3)$ diagonal matrix whose ith diagonal entry is the compliance of the ith actuator.

and finally, we obtain

$$
\Delta \mathbf{c}=\mathbf{J}_{4}\left(\mathbf{A} \mathbf{J}_{4}\right)^{-1} \mathbf{B C B}{ }^{T}\left(\mathbf{A} \mathbf{J}_{4}\right)^{-T} \mathbf{J}^{T}{ }_{4} \mathbf{W}
$$

Hence, we obtain the Cartesian compliance matrix as

$$
\mathbf{C}_{c}=\mathbf{J}_{4}\left(\mathbf{A} \mathbf{J}_{4}\right)^{-1} \mathbf{B C} \mathbf{B}^{T}\left(\mathbf{A} \mathbf{J}_{4}\right)^{-T} \mathbf{J}_{4}^{T}
$$

with

$$
\Delta \mathbf{c}=\mathrm{C}_{c} \mathbf{w}
$$

where $\mathbf{C}_{c}$ is a symmetric positive semi-definite (6x6) matrix, as expected. With this model, we can do design optimization, performance evaluation and integrated control and trajectory control. 


\section{CONCLUSIONS}

The design of reconfigurable machine tools has been addressed in the paper. Design methodology for this kind of machine tool, especially for reconfigurable parallel kinematic machine tool is discussed in detail. A case study is given to show the procedures of generating the reconfigurable parallel mechanism, and models for further design and analysis.

\section{ACKNOWLEDGEMENTS}

The author acknowledges the support for this work provided by the Natural Sciences and Engineering Research Council of Canada (NSERC) and Kayla Viegas for the CAD models.

\section{REFERENCES}

1. D. Fedewa, M. Mehrabi, S. Kota, and V. Gopalakrishnan, Design of a parallel structure ficture for reconfigurable machining systems, Proceedings of the 2000 Japan-USA Flexible Automation Conference, Ann Arbor, USA, 2000

2. Y. Koren and S. Kota, 1999, Reconfigurable machine Tools, US patent 5,943,750

3. R.G. Landers, A New Paradigm in Machine Tools: Reconfigurable Machine Tools, Japan-USA Symposium on Flexible Automation, Ann Arbor, USA, 2000

4. R.G. Landers, B.K. Min and Y. Koren, Reconfigurable Machine Tools, Annals of the CIRP, Vol.50/1, pp. 269-274, 2001

5. D. Zhang, C. Mechsfske and F. Xi, Stiffness Analysis of Reconfigurable Parallel Mechanisms with Prismatic Actuators, CIRP $1^{\text {st }}$ International Conference on Reconfigurable Manufacturing, Ann Arbor, Michigan, 2001

6. D. Zhang, C. Mechsfske and F. Xi, Optimization of Reconfigurable Parallel Mechanisms with Revolute Actuators, CIRP $1^{\text {st }}$ International Conference on Reconfigurable Manufacturing, Ann Arbor, Michigan, 2001

7. D. Zhang, Kinetostatic Analysis and Optimization of Parallel and Hybrid Architectures for Machine Tools, Ph.D. Thesis, Laval University, Quebec, 2000

8. A.S. Yigit, A.G. Ulsoy, Design of Vibration Isolation Systems for Reconfigurable Precision Equipment, Japan-USA Symposium on Flexible Automation, Ann Arbor, USA, 2000

9. Y. Koren, U. Heisel, F. Jovane, T. Moriwaki, G. Pritschow, G. Ulsoy, H. Van Brussel, Reconfigurable Manufacturing Systems, Annals of the CIRP, Vol. 48/2, 1999

10. F. Jovane, S. P. Negri, I. Fassi, L. Molinari Tosatti, Design Issues for Reconfigurable PKMs, 3th Chemnitz Parallel Kinematics Seminar: Development Methods and Application Experience of Parallel Kinematics. Chemnitz 2002 